No potential conflict of interest relevant to this letter was reported.

1. Cserti C, Haspel R, Stowell C, Dzik W. Light-chain removal by plasmapheresis in myeloma-associated renal failure. Transfusion 2007;47:511-4.

2. Bradwell AR. Serum free light chain analysis. 4th ed. Birmingham, United Kingdom: The Binding Site, 2006.

3. Hutchison CA, Cockwell P, Reid S, et al. Efficient removal of immunoglobulin free light chains by hemodialysis for multiple myeloma: in vitro and in vivo studies. J Am Soc Nephrol 2007;18: 886-95.

4. Clark WF, Garg AX. Plasma exchange for myeloma kidney: cast(s) away? Kidney Int 2008;73:1211-3.

5. Clark WF, Steward KA, Rock GA, et al. Plasma exchange when myeloma presents as acute renal failure: a randomized controlled trial. Ann Intern Med 2005;143:777-84. [Erratum, Ann Intern Med 2007;146:471.]

THE AUTHORS REPLY: The use of extracorporeal means of removing toxic immunoglobulin free light chains responsible for cast nephropathy in patients with myeloma is controversial. The largest of the three randomized trials to test plasma exchange did not show a statistically significant difference in the composite outcomes defined by the study. ${ }^{1}$ Unfortunately, this study had many limitations, including a lack of a clear clinical or histologic definition of myeloma cast nephropathy, the type of renal injury amenable to extracorporeal removal of free light chains. ${ }^{2}$ Two other smaller, randomized trials of plasma exchange suggested meaningful clinical benefit. 3,4 All three trials were done before the introduction of new agents in myeloma. In this context, given the recognized adverse impact of renal failure on survival, its potential reversibility, and the safety of the treatment procedure, we recommend additional trials.

The issue of whether an extended duration of dialysis with high-cutoff dialyzers is more effective than plasma exchange at removing free light chains or reversing renal failure is not settled. No comparative trials have been conducted to date. So far, high-cutoff dialyzers have been tested only in in vitro studies and in small uncontrolled clinical trials. Their purported effectiveness in reversing renal failure is primarily in patients treated with concurrent chemotherapy and having a response to such treatment. Further, other researchers report similar rates of reduction in levels of free light chains between plasma exchange and high-cutoff dialyzers when a standard duration of dialysis is used. ${ }^{5}$ Thus, the data are insufficient to suggest that one method is superior to the other.

In terms of the type of chemotherapy, we currently use bortezomib and dexamethasone in combination with either cyclophosphamide or thalidomide in these patients. In our letter, it was not our intention to separate the contribution of plasmapheresis from bortezomib-based therapy. We wholeheartedly agree that a formal clinical trial is needed. As Hutchison and colleagues mention, recent evidence suggests that the speed at which a reduction in levels of free light chains occurs may be as important as the amount of reduction. We hope that the high rate of renal response that we reported will inspire further studies of plasma exchange and other methods of extracorporeal removal of free light chains as adjuvant strategies in modern myeloma therapy.

Nelson Leung, M.D.

Brian L. Burnette, M.D.

S. Vincent Rajkumar, M.D.

Mayo Clinic

Rochester, MN

rajkumar.vincent@mayo.edu

Since publication of their letter, the authors report no further potential conflict of interest.

1. Clark WF, Stewart AK, Rock GA, et al. Plasma exchange when myeloma presents as acute renal failure: a randomized, controlled trial. Ann Intern Med 2005;143:777-84. [Erratum, Ann Intern Med 2007;146:471.]

2. Leung N, Gertz MA, Zeldenrust SR, et al. Improvement of cast nephropathy with plasma exchange depends on the diagnosis and on reduction of serum free light chains. Kidney Int 2008;73:1282-8.

3. Zucchelli P, Pasquali S, Cagnoli L, Ferrari G. Controlled plasma exchange trial in acute renal failure due to multiple myeloma. Kidney Int 1988;33:1175-80.

4. Johnson WJ, Kyle RA, Pineda AA, O'Brien PC, Holley KE. Treatment of renal failure associated with multiple myeloma: plasmapheresis, hemodialysis, and chemotherapy. Arch Intern Med 1990;150:863-9.

5. Hasegawa M, Kondo F, Yamamoto K, et al. Evaluation of blood purification and bortezomib plus dexamethasone therapy for the treatment of acute renal failure due to myeloma cast nephropathy. Ther Apher Dial 2010;14:451-6.

\title{
Protection against Malaria by MSP3 Candidate Vaccine
}

TO THE EDITOR: In 2007, we conducted a doubleblind, randomized, phase $1 \mathrm{~b}$ clinical trial (ClinicalTrials.gov number, NCT00452088) using the merozoite surface protein 3 (MSP3) vaccine in a malaria-endemic area. A total of 45 children who were 12 to 24 months of age were randomly as- 
signed in a 1:1:1 ratio to receive three doses (on days 0,28 , and 56) of MSP3 at a dose of $15 \mu \mathrm{g}$, MSP3 at a dose of $30 \mu \mathrm{g}$, or hepatitis B vaccine at a dose of $10 \mu \mathrm{g}$. Details of ethical approval (which included approval by the national ethics committee of Burkina Faso and written informed consent), study vaccines, safety, and immunogenicity results have been reported previously ${ }^{1}$ (see the Supplementary Appendix, available with the full text of this letter at NEJM.org). The study was sponsored by the African Malaria Network Trust through a grant from the European Commission's EuropeAid Co-operation. The MSP3 vaccine was manufactured by Synprosis, who had no role in the conduct of the study or in preparation of the manuscript.

The trial was not designed to measure vaccine efficacy. However, to monitor safety, passive surveillance of all episodes of illness, including clinical malaria, was maintained during the ensuing malaria-transmission season in a blinded fashion. Since numerous episodes of malaria were recorded, we considered examining the possibility of a protective effect induced by the MSP3 vaccine. A plan to compare the incidence of clinical malaria among the three groups was agreed on before the analysis began. Given the high transmission rate in the study area $(>200$ infected bites per person per year), ${ }^{2}$ clinical malaria was defined as a temperature of $37.5^{\circ} \mathrm{C}$ or higher in the presence of Plasmodium falciparum parasitemia at a density of 5000 parasites per microliter or more. ${ }^{3}$ The risk period analyzed was from 4 weeks after the third vaccine dose until the end of the transmission season.

The incidence rates of clinical malaria were substantially lower in each of the two groups that received the MSP3 vaccine (1.2 and 1.9 cases per 100 days, respectively), than in the group that received the hepatitis $B$ vaccine $(5.3$ cases per 100 days) $(\mathrm{P}=0.01)$ (Fig. 1). Thus, despite the small sample size and the high cumulative incidence in all groups, there was some indication that MSP3 vaccine protected against clinical malaria, at least in the short term. A sensitivity analysis with the use of a threshold density of 10,000 parasites per microliter showed broadly similar results $(\mathrm{P}=0.03)$. The similar incidence rates in the two MSP3 vaccine groups are consistent with their similar immune responses. ${ }^{1}$ These results are in keeping with the reduction in episodes of malaria associated with naturally occurring MSP3 antibodies. ${ }^{4}$

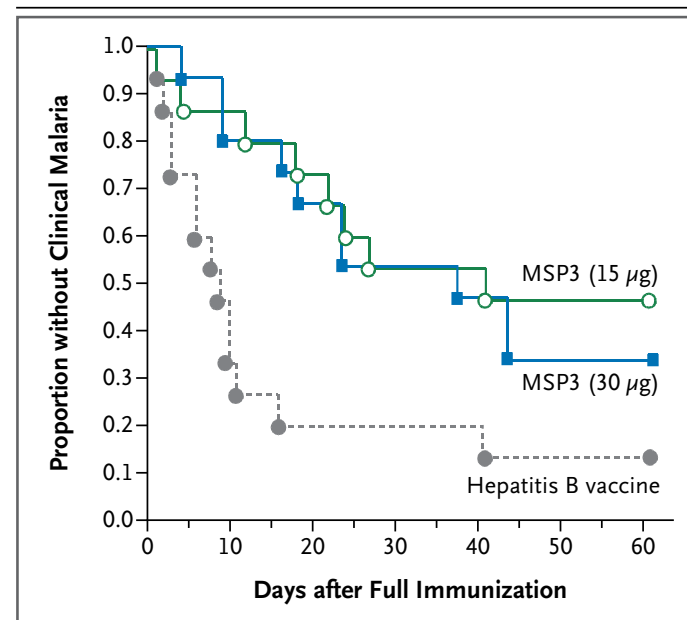

Figure 1. Kaplan-Meier Plot of the Time to the First Episode of Clinical Malaria.

There were 660, 519, and 244 days at risk (until the first episode of malaria or the end of the risk period) for the 15 patients who received MSP3 at a dose of $15 \mu \mathrm{g}$, the 15 patients who received MSP3 at a dose of $30 \mu \mathrm{g}$, and the 15 patients who received hepatitis $B$ vaccine, respectively, yielding an incidence rate of $1.2,1.9$, and 5.3 cases per 100 days, respectively. A total of 8,10 , and 13 children in these groups had an episode of clinical malaria. A log-rank test indicated evidence of a difference in the incidence rate among the three groups $(P=0.01)$. When a threshold density of 10,000 parasites per microliter was used, similar results were obtained with periods at risk of 715,563 , and 296 days, respectively, and incidence rates of $1.0,1.6$, and 4.1 cases per 100 days $(P=0.03)$. The period of analysis shown here starts after full immunization (i.e., 4 weeks after the third immunization, when antibody titers peak), and increases to the end of transmission.

It is unlikely that other efforts to control malaria influenced these results, since this was a randomized trial and the assessment of malaria was conducted in a blinded fashion. Data from the demographic surveillance system indicate that insecticide-treated bed nets were used in only 5 to $10 \%$ of households. Neither indoor insecticide spraying nor intermittent preventive treatments were practiced in the area. The study clinic provided free diagnosis and treatment 24 hours a day, so it is likely that most or all episodes of symptomatic malaria were detected. Despite the limitations in sample size and study design, we believe that the findings of this trial warrant further evaluation of this vaccine candidate.

Sodiomon B. Sirima, M.D., Ph.D.

Centre National de Recherche et de Formation sur le Paludisme Ouagadougou, Burkina Faso

s.sirima.cnlp@fasonet.bf 
Simon Cousens, M.A.

London School of Hygiene and Tropical Medicine London, United Kingdom

Pierre Druilhe, M.D.

Institut Pasteur

Paris, France

Supported by the African Malaria Network Trust through a grant from the European Commission's EuropeAid Cooperation.

Disclosure forms provided by the authors are available with the full text of this letter at NEJM.org.

1. Sirima SB, Tiono AB, Ouédraogo A, et al. Safety and immunogenicity of the malaria vaccine candidate MSP3 long synthetic peptide in 12-24 months-old Burkinabe children. PLoS ONE 2009;4(10):e7549.

2. Ilboudo-Sanogo E, Tiono BA, Sagnon N, Cuzin Ouattara N, Nébié I, Sirima SB. Temporal dynamics of malaria transmission in two rural areas of Burkina Faso with two ecological differences. J Med Entomol 2010;47:618-24.

3. Smith T, Ross A, Maire N, Rogier C, Trape JF, Molineaux L. An epidemiologic model of the incidence of acute illness in Plasmodium falciparum malaria. Am J Trop Med Hyg 2006;75:2 Suppl:56-62.

4. Roussilhon C, Oeuvray C, Muller-Graf C, et al. Long-term clinical protection from falciparum malaria is strongly associated with IgG3 antibodies to merozoite surface protein 3. PLoS Med 2007;4(11):e320.

Copyright () 2011 Massachusetts Medical Society.

\section{INSTRUCTIONS FOR LETTERS TO THE EDITOR}

Letters to the Editor are considered for publication, subject to editing and abridgment, provided they do not contain material that has been submitted or published elsewhere. Please note the following:

- Letters in reference to a Journal article must not exceed 175 words (excluding references) and must be received within 3 weeks after publication of the article.

- Letters not related to a Journal article must not exceed 400 words.

- A letter can have no more than five references and one figure or table.

- A letter can be signed by no more than three authors.

- Financial associations or other possible conflicts of interest must be disclosed. Disclosures will be published with the letters. (For authors of Journal articles who are responding to letters, we will only publish new relevant relationships that have developed since publication of the article.)

- Include your full mailing address, telephone number, fax number, and e-mail address with your letter.

- All letters must be submitted at authors.NEJM.org.

Letters that do not adhere to these instructions will not be considered. We will notify you when we have made a decision about possible publication. Letters regarding a recent Journal article may be shared with the authors of that article. We are unable to provide prepublication proofs. Submission of a letter constitutes permission for the Massachusetts Medical Society, its licensees, and its assignees to use it in the Journal's various print and electronic publications and in collections, revisions, and any other form or medium.

\section{CORRECTION}

A Problem in Gestation (September 1, 2011;365:843-8). The images shown as Figures 1 and 2 in the printed Journal (page 846) were swapped: the image for Figure 1 (the MRI scan of the abdomen) was incorrectly shown as Figure 2, and the image for Figure 2 (the ultrasonographic image of the neck) was incorrectly shown as Figure 1. The figure legends were also swapped; the figure titles were correct. Also, in the boldface paragraph beginning "After resection" (page 846), the second sentence should have read, "Maintenance doses of $600 \mathrm{mg}$ of calcium carbonate with 200 IU of vitamin D twice daily were prescribed, in addition to 400 IU of vitamin D3 daily," rather than “. . . were prescribed, with the addition of vitamin D3 (40 IU daily)." In the paragraph beginning "The approach to the management" (page 847), in the sentence beginning, "Calcitonin, which is classified," the parenthetical description of a category $\mathrm{C}$ medication for pregnant patients was incorrect; it should have read, "(i.e., a medication for which animal studies have shown an adverse effect on the fetus, but no adequate, well-controlled studies have been conducted in humans; potential benefits may warrant use of the drug in pregnant women despite potential risks)," rather than "(i.e., a medication for which there is no evidence of harm to the fetus in studies in animals but for which no adequate studies have been conducted in pregnant women)." We regret the errors. The article is correct at NEJM.org.

\section{NOTICES}

Notices submitted for publication should contain a mailing address and telephone number of a contact person or department. We regret that we are unable to publish all notices received. Notices also appear on the Journal's Web site (NEJM.org/medical-conference). The listings can be viewed in their entirety or filtered by specialty, location, or month.

\section{TH ANNUAL PRACTICING PHYSICIAN'S APPROACH TO THE DIFFICULT HEADACHE PATIENT}

The course will be offered in Rancho Mirage, CA, Feb. 17-20.

Contact Dr. Merle L. Diamond, Diamond Headache Clinic Research \& Educational Foundation, 1235a North Clybourn Ave., Suite 408, Chicago, IL 60610; or call (877) 706-6363 or (773) 883-2062; or fax (773) 883-2073; or e-mail info@dhc-fdn .org; or see http://www.dhc-fdn.org.

\section{MAYO CLINIC}

The following meetings will be held in Rochester, MN: "28th Mayo Clinic Dermatology Symposium: The O'Leary Meeting" (Sept. 23 and 24); "A Taste of Tropical Medicine: A Global Health Volunteerism Short Preparatory Course" (Oct. 28 and 29); "Geriatric Update for the Primary Care Provider" (Nov. 10); and "21st Annual Mayo Clinic Symposium on Sports Medicine" (Nov 11 and 12).

Contact the Mayo School of Continuous Professional Development, 200 First St. SW, Rochester, MN 55905; or call (800) $323-2688$ or (507) 284-2509; or fax (507) 284-0532; or see http://www.mayo.edu/cme; or e-mail cme@mayo.edu. 